\title{
Estrategias didácticas que emplean los docentes en la enseñanza de la Cinemática
}

Didactic strategies that teachers use to teach Kinematics

\author{
Osweldimar Sánchez_osweldimar35@gmail.com \\ Código ORCID: 0000-0001-5780-5780 \\ Ministerio del Poder Popular para la Educación, Venezuela
}

Recibido septiembre 2019 | Arbitrado octubre 2019 | Publicado enero 2020

\section{Resumen}

Palabras clave:

Estrategias;

enseñanza;

docentes;

cinemática

\section{Abstract}

Keywords:

Strategies; teaching; teacher; kinematics
La investigación tuvo como objetivo general analizar las estrategias didácticas que emplean los docentes en la enseñanza de la cinemática en el programa de física de 4to año. Se enmarcó en el paradigma cuantitativo, de tipo descriptivo. La población estuvo integrada por 9 docentes: 5 pertenecen a liceos públicos y 4 a liceos privados Se utilizó una encuesta tipo cuestionario conformada por 33 ítems. El instrumento fue validado mediante el juicio de expertos, arrojando un índice de confiabilidad calculado a través de Alpha de Cronbach de 0,96, mostrando una confiabilidad muy alta. La investigación reveló que se deben generar nuevas estrategias didácticas por parte de los docentes en la enseñanza de la cinemática en el programa de física de 4to año. Entre las recomendaciones de esta investigación se alcanzó que los docentes asignados a la asignatura de física sean directamente especialistas en el área.

The research has the general objective of analyzing the didactic strategies that teachers use to teach kinematics in the 4 th year physics program. The research is framed in the quantitative, descriptive type paradigm. The population consisted of 9 teachers in which 5 belonged to public lyceums and 4 to private lyceums. A questionnaire-type survey made up of 33 items was used. The instrument was validated by expert judgment, yielding a reliability index calculated using Cronbach's Alpha of 0.96 , showing very high reliability. Research reveals that new teaching strategies must be generated by teachers in teaching kinematics in the 4th year physics program. Among the recommendations of this research, it was achieved that the teachers assigned to the subject of physics are directly specialists in the área. 


\section{INTRODUCCIÓN}

La enseñanza y aprendizaje debe concebirse como un proceso organizado, coherente $\mathrm{y}$ dinámico, capaz de promover el desarrollo de habilidades y destrezas en las y los estudiantes para la apropiación significativa del conocimiento, con aplicabilidad en un contexto real. En este orden de ideas, el fin primordial de la educación en los distintos niveles, está orientado básicamente a fortalecer la capacidad de análisis, el pensamiento lógico, la creatividad, el aprendizaje por descubrimiento, la integralidad de los saberes y la construcción del conocimiento a partir de múltiples situaciones que guarden relación con el contexto sociocultural que envuelve a todos los actores del hecho educativo.

Es por ello que la Organización para la Educación, la Ciencia y la Cultura de las Naciones Unidas, UNESCO (2001), plantea que "la educación es el principal agente de transición hacia el desarrollo sostenible, pues incrementa la capacidad de las personas de hacer realidad sus concepciones de la sociedad". (s/p). En relación a estas consideraciones, la educación como proceso social integral, no debe limitarse simplemente a la transmisión de conocimientos aislados de la realidad que signa el contexto de las y los estudiantes, es necesario que, en el desarrollo de capacidades científicas y técnicas, se establezcan relaciones coherentes y no arbitrarias entre el conocimiento aprendido, el contexto, los intereses y necesidades del estudiante.

En tal sentido, el proceso educativo es permanente en la vida del ser humano, en la escuela o educación primaria se constituye la interacción con las demás personas y elementos de su entorno que le servirá para su posterior incorporación en educación media general y luego al nivel universitario y profesional, el docente debe transformarse en un mediador del proceso de enseñanza y aprendizaje, es decir, un profesional que prepara al estudiante para la vida, contribuyendo al desarrollo de competencias socio- afectivas, dando forma a la personalidad, desarrollando el pensamiento crítico y científico; además, favoreciendo la adquisición de conocimientos, hábitos, destrezas, habilidades, actitudes y valores dentro de un marco de disciplina indispensable para su formación integral.

En este orden de ideas, García (2013) considera que "el rol del docente como mediador en la construcción del conocimiento debe estar centrado en promover un conjunto de acciones que fomenten el aprendizaje significativo $y$ contextualizado en sus estudiantes" (p. 54), razón por la cual es necesario trascender del paradigma conductista que hace del docente un transmisor de información y del estudiante un receptor pasivo de ese mensaje. La educación venezolana requiere de un proceso bidireccional, más claramente, de situaciones didácticas que promuevan la interacción docente estudiante, y ambos en la realidad y el contexto sociocultural que envuelve el hecho educativo, elemento éste que ha de caracterizar un proceso educativo centrado en la aplicabilidad del conocimiento.

Frecuentemente los docentes se encuentran con barreras que les impide cumplir su propósito de lograr el aprendizaje estudiantes. Las clases tradicionales donde se instruía un tema en particular generaban poco interés, ya que también no existen laboratorios en algunos centros, así como lo es en este caso, donde realizar prácticas experimentales. Es así como los profesores señalan las distintas dificultades que presentan los estudiantes en la comprensión de contenidos de física, sin preguntarse si realmente el problema es de sus estudiantes o de las estrategias metodológicas aplicadas por ellos durante la dirección del aprendizaje de los alumnos en el proceso de enseñanza aprendizaje, en el cual tanto el docente como el estudiante son artífices y promotores de las vivencias de aprendizaje. 
Considerar la enseñanza de la física como elemento importante en el desarrollo de pensamiento científico en las aulas de clase, ha sido y seguirá siendo, uno de los pilares de la educación en el sistema educativo de nuestro país. Ha existido la creencia que la física sólo es un cúmulo de conceptos y fórmulas que funcionan a cabalidad en el desarrollo de una situación problema idealizada con la implementación de modelos matemáticos completamente abstractos.

La metodología más habitual utilizada por los docentes es la tradicional, centrada en las explicaciones teóricas del profesor y la toma de apuntes, y cuyos recursos didácticos principales son la pizarra tradicional, el libro de texto, el laboratorio e incluso la proyección de presentaciones digitales. Sin embargo, son muchos los detractores de esta metodología, ya que no permite la participación del alumno en el proceso de aprendizaje, de manera que la clase se convierte en algo tedioso y aburrido para el estudiante, que desconecta de la explicación y deja de atender. Además, la falta de interacción del alumno no permite al profesor evaluar si éste comprende o no los conceptos, y por tanto ir adaptando las explicaciones para la mejor asimilación de los contenidos.

Por otra parte, es frecuente que, por temas organizativos, en la enseñanza de la Física se separen las clases de teoría, resolución de problemas y prácticas de laboratorio, generando así, una falta de conexión entre los conceptos y las aplicaciones prácticas. Tal como afirma Franco (2010):

La separación de teoría,
problemas y prácticas es
didácticamente poco aconsejable
y bajo ningún punto de vista
viene impuesta por la estructura
de la Física, que es un cuerpo de
conocimiento compacto en el que
se conjugan aspectos teóricos y
prácticos. (...). Esta separación es
normalmente discriminatoria

para las prácticas, ya que su peso relativo disminuye frente a la teoría y los problemas. Éstos se convierten, de este modo, en el factor determinante a la hora de evaluar el rendimiento de los alumnos. (p.101)

Aun así, Palacios (2014) explica que los libros de texto han ido evolucionando para adaptarse a los cambios de la pedagogía, siendo habitual encontrar resúmenes, mapas conceptuales, preguntas para motivar la curiosidad del alumno existen dos tipos de imágenes: los modelos y las gráficas. Los modelos sirven para ilustrar descripciones y explicaciones sobre los fenómenos naturales para facilitar su comprensión. Por otro lado, las gráficas representan de forma visual la relación entre variables.

El laboratorio es uno de los recursos más utilizados por los profesores de ciencias, y resulta muy eficaz para facilitar la comprensión de los conceptos, ya que aproxima a teoría a la realidad y permite que los alumnos tomen parte activa en el aprendizaje, siendo ellos mismos quienes lleven a cabo la experiencia, observen los fenómenos y reflexionen sobre los resultados obtenidos, fomentando la autonomía.

El laboratorio desempeña una serie de funciones que refuerzan o complementan las explicaciones teóricas que realiza el profesor en clase. Caamaño (1992) manifiesta que:

tradicionalmente, los trabajos prácticos han sido utilizados como un medio para adquirir habilidades prácticas para el uso y manipulación de aparatos, para el aprendizaje de determinadas técnicas experimentales, y como una forma de ilustrar o de comprobar experimentalmente muchos de los hechos y leyes científicas presentadas previamente por parte del profesor. (p. 09) 
Sin embargo, la experiencia demuestra que el uso que se le da al laboratorio en la actualidad no distancia demasiado del que Caamaño (1992) consideraba tradicional, no ha evolucionado como lo ha hecho la enseñanza de la Física.

A pesar de que nos distancia de lo tradicional no se aplica los métodos requeridos para enseñanza. Por ello, los estudiantes poseen una falta de comprensión de conceptos en el aula de clase obteniendo así una baja motivación a la asignatura, igualmente se le dificulta los conocimientos vivenciales de muchos fenómenos y esto ayuda a que el estudiante no pueda visualizar las ilustraciones que tienen relación entre variables significativas en la interpretación de un fenómeno, sin este apoyo se les complica establecer experiencias para constatar hipótesis emitidas desde los conceptos adquiridos en el aula de clase, y por último, la falta de conocer, experimentar y desarrollar sus propias hipótesis se les complica a los aprendices realizar un grupo de trabajo para desenvolver las actividades que el docente ejecute.

Las concepciones alternativas de los alumnos son habituales en Física, y suponen un importante obstáculo para una comprensión adecuada de los fenómenos físicos, ya que la materia está compuesta por contenidos cuyos nombres son familiares en el vocabulario de los alumnos, y que por tanto generan gran cantidad de ideas previas erróneas, que resultan difíciles de erradicar, ya que se encuentran muy arraigadas por el proceder de la intuición.

Igualmente, Pozo y Gómez (2006) sostienen que estas ideas previas que los alumnos suelen utilizar para explicar la realidad de forma intuitiva, compiten con éxito con lo que se enseña en la escuela. Además, en la enseñanza de la física, se recurre a representaciones idealizadas que ayudan al alumno a simplificar los problemas que se plantean, para poder profundizar en ellos y llegar a comprenderlos, pero que están bastante alejadas de la realidad que percibe.
Por otra parte, también se debe tener en cuenta las ideas previas insuficientes que limitan la comprensión de nuevos conceptos basados en los aprendizajes anteriores. Muchas veces, los alumnos llegan a la etapa de Bachillerato con deficiencias en varios conceptos que se consideran asimilados y que son necesarios para poder seguir avanzando en la materia. Es habitual que estas ideas previas erróneas o insuficientes se deban a tareas repetitivas, en las que el alumno reproduce las respuestas de forma mecánica sin razonar, o basándose en la intuición más que en razones científicas.

Se puede estimar que durante los trabajos prácticos las principales dificultades de los laboratorios no motivan a los alumnos, no les están brindando la oportunidad de trabajar de forma autónoma, y a menudo, en grupo, siendo el trabajo en grupo una motivación por sí mismo, debido a la interacción con los compañeros. El factor motivador que añade el laboratorio está relacionado con el papel protagonista en que sitúa al alumnado, haciéndoles partícipes del método científico y aproximando la ciencia a su realidad.

Aunque, al margen de todas estas ventajas, también se encuentran muchos los autores que se posicionan en contra de los trabajos prácticos o destacan sus limitaciones. Carvajal y Gómez, (2002) señalan que:

$$
\begin{aligned}
& \text { Los maestros tienen poca } \\
& \text { confianza en el nivel de análisis al } \\
& \text { que pueden llegar sus alumnos; la } \\
& \text { mayoría se queja del tipo de } \\
& \text { conclusiones que expresan en los } \\
& \text { reportes de las prácticas de } \\
& \text { laboratorio o de la dificultad de } \\
& \text { entablar una discusión y reflexión } \\
& \text { en grupo. (p. 65) }
\end{aligned}
$$

Con todo, se estima que la Cinemática no tiene un papel relevante en los laboratorios, las prácticas de Cinemática que suelen realizarse en el Liceo Nacional de Venezuela en Edo. Carabobo 
Municipio Bejuma se limitan a medir tiempos y distancias en movimientos simples. Además de las limitaciones espaciales y los problemas de visualización, existe una gran dificultad de medida para movimientos más complejos, como podría ser un tiro parabólico, en el que no podrían estimarse ni ángulos, ni alturas, ni parámetros instantáneos (velocidad y aceleración instantáneas).

Al respecto, Suárez (2001) expresa que "la Física es una ciencia, que se ocupa de los componentes fundamentales del universo, de las fuerzas que éstos ejercen entre sí y de los efectos de dichas fuerzas" (p. 89), por lo tanto desde tiempo atrás muchas personas se han interesado por la enseñanza de las ciencias y en particular de la física, de allí radica toda la problemática existente para con la enseñanza y el aprendizaje de la física en cualquier nivel de instrucción, puesto que saber cómo enseñar ciencias es, lógicamente, uno de los cometidos del profesorado encargado de esta disciplina.

No obstante, la didáctica del docente que administra la enseñanza de Física, en los momentos presentes, evidencia notables carencias en cuanto a la aplicación de estrategias para la enseñanza en el aprendizaje significativo de los contenidos inherentes a esta ciencia. Se evidencia poca comprensión de elementos conceptuales y procedimentales relacionados con tópicos de Física, además de bajo rendimiento académico en esta importante área del conocimiento científico universal, cuestión que repercute de forma negativa en la formación integral de los estudiantes.

En correspondencia con lo planteado, es necesario resaltar que la poca apropiación del conocimiento de la física por parte de los estudiantes, está íntimamente relacionada con la praxis pedagógica del docente, pues el desarrollo de los contenidos se centra básicamente en la memorización de teorías y ecuaciones sin comprensión y aplicabilidad real. Al respecto, González (2012) afirma que existen:
Notables dificultades en cuanto a las estrategias aplicadas por los docentes al momento de desarrollar los contenidos propios de esta Ciencia, específicamente se hace referencia a la falta de contextualización del aprendizaje en situaciones y tiempo real, pues la solución a los problemas propios de la Física impartidos en los distintos niveles educativos, se reduce simplemente a la aplicación de fórmulas y algoritmos, no se evidencia el diseño y aplicación de estrategias que fomenten la construcción del conocimiento por parte de los estudiantes partiendo de casos particulares hasta el establecimiento de relaciones generales, en escasas oportunidades se hace uso del laboratorio y con él, la aplicación del método científico experimental para demostrar el comportamiento de los cuerpos o hechos físicos estudiados en el aula de clase. (p. 145)

Cabe destacar que la enseñanza de la ciencia y particularmente de la Física, debe estar asignada por el diseño y ejecución de actividades creativas, motivadoras $\mathrm{y}$ ejemplarizantes, por lo que es necesario considerar los conocimientos previos de las y los estudiantes, hecho que permite construir de forma proactiva el conocimiento general. Sin embargo, es evidente que el docente durante su praxis pedagógica se auto limita, generando obstáculos didácticos que inciden considerablemente en la construcción y apropiación del conocimiento por parte de las y los estudiantes.

Aunado a esto, Rodríguez (2013) establece "que la construcción, contextualización y aplicabilidad del conocimiento Físico, logra minimizar los obstáculos que se presentan los estudiantes durante la apropiación del aprendizaje de la Física" (p. 25). Razón por la cual el docente durante su praxis pedagógica debería entender que la Física es una ciencia 
abstracta, pero íntimamente relacionada con la cotidianidad del aprendiz, por lo que cada estrategia didáctica debe orientarse hacia la construcción significativa del conocimiento, en contraposición con el estilo conductista de la enseñanza tradicional, en la cual se evidencia memorización excesiva y bajos niveles de aprendizaje.

Es importante destacar que en muchas ocasiones el docente no cuenta con material didáctico que promueva aprendizaje significativo y contextualizado, los textos de Física han sido elaborados para lo general, por consiguiente, es responsabilidad del docente adaptarlos a situaciones reales propias del ambiente y contexto escolar. No obstante, tal adaptación es ligeramente delicada, pues requiere de la investigación y análisis continúo por parte de los docentes, exige además el diseño de estrategias que, sin eludir la cientificidad del conocimiento científico universal, sean capaces de promover la construcción del aprendizaje por parte de los estudiantes con la mediación del docente.

Por otro lado, la mayoría de los docentes de educación secundaria del Municipio Bejuma promueven un proceso de enseñanza $\mathrm{y}$ aprendizaje de esta ciencia bajo un enfoque axiomático, es decir, se parte de fórmulas, teorías y leyes preestablecidas, las cuales por lo general son memorizadas por los estudiantes sin ningún significado real. En relación a este aspecto, es importante resaltar que el rol del docente como mediador en la construcción del conocimiento, debe estar dirigido a fomentar la comprensión de las terminologías, los conceptos físicos y la naturaleza de las ecuaciones o fórmulas matemáticas que son empleadas en la resolución de los problemas, razón por la cual se hace necesario considerar situaciones particulares con tratamientos científicos, que permitan a los estudiantes participar activamente en la construcción del conocimiento general. Al respecto Vargas (2009) plantea que:
El razonamiento inductivo constituye un aspecto básico en la enseñanza y aprendizaje de las diversas áreas académicas y su productividad y eficacia surge cuando el asunto estudiado se presenta por medio de casos particulares, sugiriéndose que se descubra el principio general que los rige, este método genera gran actividad mental en las $y$ los estudiantes, involucrándolos plenamente en su proceso de aprendizaje. (p.125)

Sin embargo, la realidad actual evidencia serias dificultades en la praxis pedagógica. Especialmente el docente para presentar los contenidos de Física a través de procesos de inducción, carece de estrategias que promuevan la construcción del conocimiento general partiendo de casos particulares o hechos tangibles asociados a la realidad de las y los estudiantes. La enseñanza de la Física, en educación media general, tiende a fomentar la memorización de fórmulas y el simple cálculo numérico en la resolución de ejercicios, no se promueve una didáctica centrada en los procesos.

Sin embargo, se debe considerar la variedad de recursos con los que se cuentan hoy en día para crear estrategias metodológicas tendientes a una apropiación de elementos esenciales en el estudio de la física, en este caso reconociendo la importancia que generar en los estudiantes de Venezuela Edo. Carabobo de 4to. Año de la Educación Media General una mejor interpretación de fenómenos físicos asociados al aprendizaje significativo de la cinemática, es la parte de la Física que estudia la descripción del movimiento de los cuerpos.

Por lo general, en el estudio de la cinemática los estudiantes encuentran por primera vez aplicaciones del álgebra y del concepto de función, temas que son estudiados en el territorio circuital de Bejuma número 2 $\mathrm{y}$, por tanto, es importante que esta 
experiencia sea positiva y significativa para ellos, algo que sólo es posible si hay claridad en los conceptos. La cinemática también es una primera aproximación al estudio de los cambios que experimenta un sistema físico que, partiendo de un estado inicial, después de unos procesos, llega a un estado final.

Se estima destacar que, en la asignatura de física pareciera existir una situación que no conlleva a reflexionar sobre la problemática en la calidad actual de la enseñanza de dicha asignatura, ya que es de hacer notar que la enseñanza de la física debe ser fuente real de desarrollo de cualidades para la actividad cognoscitiva, tales como la independencia, la creatividad y la libre iniciativa. En este orden, Martínez (2008) plantea que:

Para aprender, el estudiante debe estar motivado, para ello es necesario conectar el tópico con los temas de interés del alumno e incitarle a hacerse preguntas, hacer de la actitud positiva del profesor un modelo para que ellos se motiven, limitar la cantidad y complejidad de estímulos, focalizar la exposición en punto de interés del educando. (p. 78)

Es importante resaltar que el contenido temático de este saber se enfoca en la cinemática, la cual estudia el movimiento de los cuerpos sin importar las causas que lo produzcan, siendo este contenido el más pertinente por relacionarse con las diferentes actividades de caída libre. La cinemática debería de tener una gran importancia en los estudiantes del territorio circuital de Bejuma, por tal motivo de ahí que se hace necesario hacer una indagación acerca de las estrategias que se desarrollan en los ambientes de aprendizaje.

Es necesario que se promueva en los ambientes escolares el razonamiento abstracto, la creatividad, el pensamiento analítico y crítico de los principales actores del hecho educativo, aspectos indispensables para que los mismos ejerzan una función socialmente útil tal como la comunidad espera que ocurra, pero además sean capaces de comprender la complejidad del conocimiento científico. Razón por la cual es imprescindible fomentar una investigación capaz de dar respuesta a la siguiente interrogante íntimamente relacionada con el problema planteado. ¿Qué estrategias didácticas que emplean los docentes en la enseñanza de la cinemática en el programa de Física de 4to año del territorio circuital de Bejuma numero 2?

En función a la pregunta realizada anteriormente se formuló el siguiente objetivo general: Analizar las estrategias didácticas que emplean los docentes en la enseñanza de la cinemática en el programa de física de 4to año en el territorio cirtuital número 2 de Bejuma. Por consiguiente para dar respuesta al objetivo general de la investigación se estructuraron los siguientes objetivos específicos: 1 . Diagnosticar las estrategias didácticas que emplean los docentes a través de la planificación en el programa de física de 4to año en el territorio circuital número 2 Bejuma; 2. Determinar las estrategias didácticas de enseñanza en el programa de ejecución en física de 4to año en el territorio circuital número 2 Bejuma; 3. Identificar las estrategias didácticas que emplean los docentes en la evaluación de la cinemática en el programa de física de 4to año en el territorio circuital número 2 Bejuma.

Luego de haber reducido el problema a términos precisos y explícitos, es decir, restringido y delimitado a una dimensión manejable, se procedió a darle un enfoque lo suficientemente amplio para comprender con mayor concreción la problemática planteada, se consideró pertinente y necesario situar el marco teórico o referencial que orienta el estudio en todos sus aspectos, la fundamentación teórica.

Los autores antes mencionados consideran que es importante el aprendizaje 
significativo para los estudiantes en la materia de física por medio de estrategias didácticas que empleen los docentes en el aula de clase. En las investigaciones explican que los tutores de dicha mención no aplican estrategias didácticas correctas para el aprendizaje significativo en los estudiantes, por este motivo muchos investigadores han realizado planes de estrategias didácticas, propuestas didácticas, guía de estrategias para el razonamiento inductivo, todo con el objetivo de mejorar el desempeño de los formativos y constituir un desarrollo significativo para los discípulos. Debido a que el avance obtenido dependerá del instructor al motivar a los educandos para un buen proceso de conocimiento.

\section{MÉTODO}

Los aspectos relativos a la metodología que se empleó para realizar el presente estudio son el tipo de investigación, diseño, población y muestra, así como también, se describen las técnicas e instrumentos de recolección de los datos, los procedimientos que se emplearón para dar validez y confiabilidad al instrumento que se diseñó de acuerdo a la tabla de operacionalización, y finalmente la técnica empleada para el proceso y análisis de los resultados, a fin de obtener las debidas conclusiones que dieron respuestas a los objetivos planteados.

En el momento de resolver ejercicios científicos se debe tener en cuenta un conocimiento minucioso de los diferentes tipos de investigación que se pueden alcanzar. Esta indagación hace posible impedir equivocaciones en la selección del método conveniente para una forma específico para un producto. De acuerdo, con esto la presente disertación es de campo donde Tamayo y Tamayo (2009), expresan lo siguiente:

Cuando se va a resolver un problema en forma científica, es muy conveniente tener un conocimiento detallado de los posibles tipos de investigación que se pueden seguir. Este conocimiento hace posible evitar equivocaciones en la elección del método adecuado para un procedimiento específico. Conviene anotar que los tipos de investigación difícilmente se presentan puros; generalmente se combinan entre sí y obedecen sistemáticamente a la aplicación de la investigación". (p. 37)

Es por ello que el tipo de investigación en el presente estudio fue de campo, dado que se llevó a cabo en el territorio circuital Bejuma donde se encontró el objeto de estudio y los datos serán recopilados directamente de la realidad.

Por su parte, el referirse al nivel de la búsqueda, se enfocó en el descriptivo. Explicado por Hernández (2012) se refiere a aquellas disertaciones "que buscan especificar las propiedades, las características y los perfiles de personas, grupos, comunidades, procesos, objetos o cualquier otro fenómeno que se someta a un análisis" (p. 102)

En tal sentido, el estudio descriptivo es aquel nivel donde el investigador va a realizar un análisis de los hechos que se observa por medio de descripción, registro, análisis a través de ellos por medio de estos se obtiene resultados que son favorables para la investigación.

En virtud de lo anterior, la presente indagación es de campo descriptivo la cual va dirigida a describir Estrategias Didácticas que emplean los Docentes en la Enseñanza de la Cinemática en el Programa de Física de 4to año en el territorio circuital número 2 Bejuma.

El presente estudio se enmarcó en el diseño no experimental, pues fue una investigación que se realizó sin manipular deliberadamente variables. 
En este sentido, para la presente disertación titulada estrategias didácticas que emplean los docentes en la enseñanza de la cinemática en el programa de física de 4to año en el municipio Bejuma, estado Carabobo, se tomará como población a los nueve (9) docentes de las referidas instituciones quienes se encargan de impartir la enseñanza de la asignatura física para el año escolar 2018-2019.

Por todo lo anterior, Palella y Martins (2012) afirman que la muestra "representa un subconjunto de la población, accesible y limitado, sobre el que realizamos las mediciones o el experimento con la idea de obtener conclusiones generalizables a la población" ( $p$. 105). Tomando en cuenta lo expuesto por el autor antes mencionado, se tomó una muestra representativa de los docentes de 4to año en el municipio Bejuma, estado Carabobo, conformado por el $90 \%$ de la población de docentes, ya que el $10 \%$ restante de docentes serán para la confiabilidad del instrumento, el cual equivale a 4 docentes que se encargan de impartir la asignatura de física en el municipio Bejuma, estado Carabobo durante el año escolar 2018-2019 en el nivel de 4to año.

\section{RESULTADOS}

El no saber cómo manejar los docentes de manera óptima los conceptos que están relacionados a la cinemática genera ciertas dificultades en los estudiantes de bachillerato a la hora de resolver complicaciones relacionados a esta, además de que la misma sirve de base fundamental en el estudio de la dinámica y de la física, en cierto modo la cinemática se imparte por primera vez en 3er año de bachillerato y en es importante que por esta razón las estrategias utilizadas sean las más acordes para que los estudiantes puedan obtener un aprendizaje significativo y la experiencia sea positiva dentro del aula o en el laboratorio, pero para esto debe haber una claridad en diversos términos básicos, dependiendo de la claridad con la cual le ejerza el docente hacia el estudiante con respecto a los términos se verá la motivación, interés, desempeño y rendimiento de cada uno de ellos en Física.

De igual manera la educación es un proceso que no solamente tiene como objetivo dar herramientas desde el punto de vista del conocimiento, también debe garantizar la formación de profesionales íntegros, reflexivos, críticos, capaces de buscar soluciones a diversas situaciones, sin importar el entorno donde se desenvuelvan los problemas, todas las sociedades requieren de ciudadanos pensantes que sean capaces de ser proactivos $y$ productivos para la misma, por lo cual construir una base sólida que permita desarrollar un pensamiento plural y libre, capaz de ver mucho más allá de lo superficial e incentivando a las personas a debatir, compartir e intercambiar conocimientos.

Por lo cual es importante tener en cuenta que el desarrollo de las clases no debe ser solamente un hecho en el cual los estudiantes obtengan una calificación para aprobar una asignatura, por el contrario debe brindar esas herramientas para que los futuros profesionales del país puedan seguir transmitiendo cada una de ellas en el futuro e incluso innovando a través de ellas.

Por lo que la investigación revela que se deben generar nuevas estrategias didácticas por parte de los docentes en la enseñanza de la cinemática en el programa de física de 4to año del territorio circuital de Bejuma número 2.

\section{CONCLUSIONES}

En función de los resultados obtenidos, se concluyó dentro de la investigación que el personal docente encuestado debe establecer una mesa de trabajo en la cual se discutan cuáles podrían ser las mejores estrategias a tratar con los estudiantes según el año siendo esto uno de los mayores factores que pueden influir en el aprendizaje significativo a los 
estudiantes, se deben establecer propuesta tanto en el docente asignado a cada área como los coordinadores pedagógicos, docentes encargado del departamento de evaluación entre otros en pro de mejorar las estrategias, no solo para la enseñanza de la Física, sino también para otras asignaturas de la institución.

Se requiere que los docentes asignados a la asignatura de física sean directamente especialistas o egresado de dicha asignatura por lo que al no ser de la asignatura no contaría con las mismas estrategias o el mismo contenido eficiente para que los estudiantes lo comprendan mejor. Podría mejorar el objetivo de que al estudiante le llegue a gustar dicha disciplina y adecuarla a los espacios que corresponde en la institución como lo son los laboratorios.

\section{REFERENCIAS}

Caamaño, A. (1992). Los Trabajos Prácticos en Ciencias Experimentales. Aula de innovación educativa, 9, 61- 68

Carvajal, E. y Gómez, M. (2002). Concepciones y Representaciones de los Maestros de Secundaria y Bachillerato sobre la Naturaleza, el Aprendizaje y la Enseñanza de las Ciencias. Revista Mexicana de Investigación Educativa, 7 (16), 577-602. Recuperado de http://campus.fundec.org.ar/admin/archi vos/ Concepciones\% 20y\%20representaciones\%20de\%20los\%20 maestros.pdf

Franco, A. (2010). La Enseñanza Tradicional. El curso interactivo de Física en Internet. Recuperado

de http://www.sc.ehu.es/sbweb/fisica/Introd uccion /física/fisica2 .htm

García, M. (2013). Evaluación de las Estrategias de Enseñanza-Aprendizaje Utilizadas por los Docentes para generar Aprendizaje Significativo en el Desarrollo del (Tema) "Movimiento Parabólico"

González, J. (2012). Magnitudes Físicas. Recuperado el 20 de enero de 2016, Recuperado de http://es.slideshare.net/dinoflagelado/ma gnitudesfisicas- 1273816

Martínez, F. (2008). Estrategias Didácticas de la física. Revista de Educación. Universidad Simón Bolívar: Caracas

Organización para la Educación, la Ciencia y la Cultura de las Naciones Unidas, UNESCO. (2001). Educación para todos en América Latina y el Caribe. Cochabamba, Bolivia

Palacios, A. (2014). Recursos Didácticos de la Especialidad. Material no publicado. Recuperado de http://msec.unir.net/cursos/msec_ per21_fq_rd/?ag=\&idtarea $=$

Palella, S. y Martíns, F. (2012). Metodología de la Investigación Cuantitativa. FEDUPEL. Fondo Editorial de la Universidad Pedagógica Experimental Libertador. La Editorial Pedagógica de Venezuela: Caracas

Pozo, I. y Gómez, M. (2006). Capítulo I: ¿Por qué los Alumnos no Aprenden la Ciencia que se les Enseña?. (5 $\left.5^{\mathrm{a}} \mathrm{Ed}.\right)$, Aprender y enseñar ciencia. Del conocimiento cotidiano al conocimiento científico (pp. 17- 32). Madrid: Morata

Rodríguez, B. (2013). ¿Por qué hacer Ciencia (básica) en Colombia?: la visión muy personal de un "científico" colombiano. Recuperado de http://www.scielo.org.co/scielo.php?scrip $\mathrm{t}=\mathrm{sci} \_$arttext\&pid=S0123779920130001070001.

Suárez, W. (2001). Teoría y Práctica de Física. Tercer año. Distribuidora Discolar: Caracas Tamayo y Tamayo. (2009). Técnicas de Investigación. México: Me Graw Hill

Vargas, I. (2009). Razonamiento Inductivo. Recuperado de prezi.com/sjirpmyu6aeu 